\title{
Multiple periodic solutions to a class of nonautonomous second-order delay differential equation
}

Qiong Meng ${ }^{1 *}$ and Xiaosheng Zhang ${ }^{2}$

\author{
"Correspondence: \\ mengqiong@qq.com \\ 'school of Mathematical Science, \\ Shanxi University, Wucheng Road, \\ Taiyuan, 030006, P.R. China \\ Full list of author information is \\ available at the end of the article
}

\begin{abstract}
The existence of the nontrivial periodic solutions for nonautonomous second-order delay differential equation

$$
x^{\prime \prime}(t)+\lambda x(t)=-f(t, x(t), x(t-\tau))
$$

is investigated, where $\lambda>\pi^{2} / \tau^{2}, \tau>0, f \in C^{1}\left(\mathbf{R} \times \mathbf{R}^{2}, \mathbf{R}\right)$. Multiple periodic solutions are obtained by some recent critical point theorems.
\end{abstract}

MSC: 34K13; 34K18; 58E50

Keywords: second-order delay differential equation; nonautonomous; periodic solution; critical point theorems

\section{Introduction}

It is well known that the critical point theory is a powerful tool to deal with the multiplicity of periodic solutions to ordinary differential systems as well as partial differential equations (see [1-6]). In 1998, Li and He [7] first applied the critical point theory to study the multiplicity of periodic solutions for delay differential equations. Especially, in 2005, Guo and $\mathrm{Yu}$ [8] established a variational framework for delay differential autonomous systems. In the past several years, some results on the existence of periodic solutions for the functional differential equation have been obtained by the critical point theory (see [7-14]). However, most of these functional differential equations are autonomous, the results on the non-autonomous functional differential equations are relatively few (see [12,13]).

Motivated by the work of $[11,12]$, we consider a class of nonautonomous second-order delay differential equation

$$
x^{\prime \prime}(t)+\lambda x(t)=-f(t, x(t), x(t-\tau))
$$

where $\lambda>\pi^{2} / \tau^{2}, \tau>0, f \in C^{1}\left(\mathbf{R} \times \mathbf{R}^{2}, \mathbf{R}\right)$.

In this paper, we have the following conditions on $f$.

$(f 1)$

$$
\begin{gathered}
f(t+\tau, x)=f(t, x), f(t,-x,-y)=-f(t, x, y) \text { and } \\
\frac{\partial f(t, x, y)}{\partial y}=\frac{\partial f(t, y, x)}{\partial x}
\end{gathered}
$$

@2013 Meng and Zhang; licensee Springer. This is an Open Access article distributed under the terms of the Creative Commons Attribution License (http://creativecommons.org/licenses/by/2.0), which permits unrestricted use, distribution, and reproduction in any medium, provided the original work is properly cited. 
for all $x, y \in \mathbf{R}, t \in[0, \tau]$

(f2) there exist four $\tau$-periodic and continuous functions $a(t), b(t), c(t)$ and $d(t)$ such that

$$
f(t, x, y)=a(t) x+b(t) y+o(r) \quad \text { as } r=\sqrt{x^{2}+y^{2}} \rightarrow 0
$$

and

$$
f(t, x, y)=c(t) x+d(t) y+o(r) \quad \text { as } r=\sqrt{x^{2}+y^{2}} \rightarrow \infty
$$

uniformly for $t \in[0, \tau]$;

$\left(f 3^{ \pm}\right)|\nabla F(t, z)-B(t) z|$ is bounded for all $z=(x, y) \in \mathbf{R}^{2}, t \in[0, \tau]$ and

$$
F(t, z)-\frac{1}{2}(B(t) z, z) \rightarrow \pm \infty \quad \text { as }|z| \rightarrow \infty
$$

uniformly for $t \in[0, \tau]$, where

$$
\begin{aligned}
& F(t, x, y)=\int_{0}^{x} f(t, s, y) d s+\int_{0}^{y} f(t, s, 0) d s, \\
& A(t)=\left(\begin{array}{ll}
a(t) & b(t) \\
b(t) & a(t)
\end{array}\right), \quad B(t)=\left(\begin{array}{ll}
c(t) & d(t) \\
d(t) & c(t)
\end{array}\right) .
\end{aligned}
$$

Theorem 1.1 Assume that $f$ satisfies $(f 1)-\left(f 3^{-}\right)$with $(A(t) z, z) \leq 0,(B(t) z, z)>(\lambda-$ $\left.\pi^{2} / \tau^{2}\right)|z|^{2}>0$ for $z \in \mathbf{R}^{2} \backslash\{0\}, t \in[0, \tau]$. Then (1.1) possesses at least $2 m$ pairs $2 \tau$-periodic solutions, where

$$
m=\max \left\{j \in \mathbf{Z}^{+}:(B(t) z, z)>\left(\lambda-\frac{\pi^{2}}{\tau^{2}} j^{2}\right)|z|^{2}>0 \text { for } z \in \mathbf{R}^{2} \backslash\{0\}, t \in[0, \tau]\right\},
$$

$\mathbf{Z}^{+}$is the set of all positive integers.

Theorem 1.2 Assume that $f$ satisfies $(f 1)-\left(f 3^{+}\right)$with $(A(t) z, z) \geq 0,(B(t) z, z)<(\lambda-$ $\left.\pi^{2} m_{0}^{2} / \tau^{2}\right)|z|^{2}<0$ for $z \in \mathbf{R}^{2} \backslash\{0\}, m_{0} \in \mathbf{Z}^{+}, t \in[0, \tau]$. Then (1.1) possesses at least $2\left[m_{1}-\right.$ $\left.m_{0}+1\right]$ pairs $2 \tau$-periodic solutions, where

$$
m_{1}=\max \left\{j \in \mathbf{Z}^{+}:(B(t) z, z)<\left(\lambda-\frac{\pi^{2}}{\tau^{2}} j^{2}\right)|z|^{2}<0 \text { for } z \in \mathbf{R}^{2} \backslash\{0\}, t \in[0, \tau]\right\} .
$$

In this paper, the main purpose is to study the multiplicity of periodic solutions for systems (1.1) via some recent critical point theorems for strongly indefinite functionals. In order to achieve this, some preliminaries are necessary. Let $X$ and $Y$ be Banach spaces with $X$ being separable and reflexive, and set $E=X \oplus Y$. Let $\mathcal{S} \subset X^{n}$ be a dense subset. For each $s \in \mathcal{S}$, there is a semi-norm on $E$ defined by

$$
p_{s}: E \rightarrow \mathbf{R}, \quad p_{s}(u)=|s(x)|+\|y\| \quad \text { for } u=x+y \in X \oplus Y .
$$

We denote by $\mathcal{T}_{\mathcal{S}}$ the topology on $E$ induced by the semi-norm family $\left\{p_{s}\right\}$, and let $\omega$ and $\omega^{*}$ denote the weak-topology and weak"-topology, respectively. Clearly, the $\mathcal{T}_{\mathcal{S}}$ topology 
contains the product topology on $E=X \oplus Y$ produced by the weak topology on $X$ and the strong topology on $Y$.

For a functional $\Phi \in C^{1}(E, \mathbf{R})$, we write $\Phi_{a}=\{u \in E: \Phi(u) \geq a\}$. Recall that $\Phi^{\prime}$ is weakly sequentially continuous if $u_{k} \rightarrow u$ in $E$, one has $\lim _{k \rightarrow \infty} \Phi^{\prime}\left(u_{k}\right) v \rightarrow \Phi^{\prime}\left(u_{k}\right) v$ for each $v \in E$, i.e., $\Phi^{\prime}:(E, \omega) \rightarrow\left(E^{*}, \omega^{*}\right)$ is sequentially continuous. For $c \in \mathbf{R}$, we say that $\Phi$ satisfies the $(C)_{c}$ condition if any sequence $\left\{u_{k}\right\} \subset E$, such that $\Phi\left(u_{k}\right) \rightarrow c$ and $\left(1+\left\|u_{k}\right\|\right) \Phi^{\prime}\left(u_{k}\right) \rightarrow 0$ as $k \rightarrow \infty$, contains a convergent subsequence.

Suppose that

$\left(\Phi_{0}\right) \Phi \in C^{1}(E, \mathbf{R}), \Phi_{c}$ is $\mathcal{T}_{\mathcal{S}}$-closed for every $c \in \mathbf{R}$ and $\Phi^{\prime}:\left(\Phi_{c}, \mathcal{T}_{\mathcal{S}}\right) \rightarrow\left(E^{*}, \omega^{*}\right)$ is continuous;

$\left(\Phi_{1}\right)$ there exists $\rho>0$ such that $\kappa:=\inf \Phi\left(B_{\rho} \cap Y\right)>0=\Phi(0)$, where $B_{\rho}=\{u \in E:\|u\|=$ $\rho\}$;

$\left(\Phi_{2}\right)$ there exist a finite dimensional subspace $Y_{0} \subset Y$ and $R>\rho$ such that $\bar{c}:=\sup \Phi\left(E_{0}\right)<$ $\infty$ and $\sup \Phi\left(E_{0} \backslash S_{0}\right)<\inf \Phi\left(B_{\rho} \cap Y\right)$, where $E_{0}:=X \oplus Y_{0}$, and $S_{0}=\left\{u \in E_{0}:\|u\| \leq R\right\}$.

The following critical point theorem will be used later (see $[6,14])$.

Theorem A Assume that $\Phi$ is even and $\left(\Phi_{0}\right)-\left(\Phi_{2}\right)$ are satisfied. Then $\Phi$ has at least $m=$ $\operatorname{dim} Y_{0}$ pairs of critical points with critical values less than or equal to $\bar{c}$ provided $\Phi$ satisfies the $(C)_{c}$ condition for all $c \in[\kappa, \bar{c}]$.

\section{Preliminaries}

In this section, we establish a variational structure which enables us to reduce the existence of $2 \tau$ periodic solutions of (1.1) to a classic Hamiltonian system. First, we have the following lemma by using similar arguments [11].

Lemma 2.1 Suppose that $f \in C^{1}\left(\mathbf{R} \times \mathbf{R}^{2}, \mathbf{R}\right)$ satisfies $(f 1)$, then the function

$$
H(t, x, y)=\int_{0}^{x} f(t, s, y) d s+\int_{0}^{y} f(t, s, 0) d s
$$

satisfies

$$
\frac{\partial H(t, x, y)}{\partial x}=f(t, x, y), \quad \frac{\partial H(t, x, y)}{\partial y}=f(t, y, x)
$$

and $H(t, x, y) \in C^{2}\left(\mathbf{R} \times \mathbf{R}^{2}, \mathbf{R}\right)$.

Proof It is obvious that $\frac{\partial H}{\partial x}=f(t, x, y)$. By $(f 1), \frac{\partial f(t, x, y)}{\partial y}=\frac{\partial f(t, y, x)}{\partial x}$. Then we have

$$
\frac{\partial H(t, x, y)}{\partial y}=\int_{0}^{x} \frac{\partial f(t, y, s)}{\partial s} d s+f(t, y, 0)=f(t, y, x) .
$$

The proof of Lemma 2.1 is complete.

Suppose that $x$ is a periodic solution of (1.1) with $2 \tau$, let $y(t)=x(t-\tau)$, then

$$
y^{\prime \prime}(t)=-f(t-\tau, x(t-\tau), x(t))=-f(t, y(t), x(t)) .
$$


We denote $z=(x, y)$. Then

$$
z^{\prime \prime}(t)+\lambda z(t)=-\nabla H(t, z(t))
$$

where $H(t, z)$ is defined in (2.1) and $\nabla H(t, z)$ denotes the gradient of $H(t, z)$ with respect to the $z$ variable. It is easy to obtain the following lemma.

Lemma 2.2 For any $2 \tau$ periodic solution of $(1.1)$, let $y(t)=x(t-\tau)$, then $z=(x, y)$ is a $2 \tau$ periodic solution of (2.2). Conversely, for any $2 \tau$ periodic solution $z=(x, y)$ of $(2.2)$ satisfying $y(t)=x(t-\tau), x$ is a $2 \tau$ periodic solution of $(1.1)$.

For $S^{1}=\mathbf{R} /(2 \tau \mathbf{Z})$, let $C^{\infty}\left(S^{1}, \mathbf{R}^{2}\right)$ denote the space of $2 \tau$ periodic $C^{\infty}$ functions on $\mathbf{R}$ with values in $\mathbf{R}^{2}$. For any $z \in C^{\infty}\left(S^{1}, \mathbf{R}^{2}\right)$, it has the following Fourier expansion in the sense that it is convergent in the space $L^{2}\left(S^{1}, \mathbf{R}^{2}\right)$,

$$
z(t)=\frac{a_{0}}{\sqrt{2 \tau}}+\frac{1}{\sqrt{\tau}} \sum_{j=1}^{\infty}\left[a_{j} \cos \left(\frac{\pi}{\tau} j t\right)+b_{j} \sin \left(\frac{\pi}{\tau} j t\right)\right]
$$

where $a_{0}, a_{j}, b_{j} \in \mathbf{R}^{2}, j=1,2, \ldots$

Let $z \in L^{2}\left(S^{1}, \mathbf{R}^{2}\right)$. If there exists a function $y \in L^{2}\left(S^{1}, \mathbf{R}^{2}\right)$ such that, for every $x \in$ $C^{\infty}\left(S^{1}, \mathbf{R}^{2}\right)$

$$
\int_{0}^{2 \tau}\left(z(t), x^{\prime}(t)\right) d t=-\int_{0}^{2 \tau}(y(t), x(t)) d t
$$

then $y$ is called a weak derivative of $z$ denoted by $y=z^{\prime}(t)$.

Let

$$
H^{1}\left(S^{1}, \mathbf{R}^{2}\right)=\left\{z \in L^{2}\left(S^{1}, \mathbf{R}^{2}\right) \mid \int_{0}^{2 \tau}\left(|z(t)|^{2}+\left|z^{\prime}(t)\right|^{2}\right) d t<+\infty\right\} .
$$

For any $z, y \in H^{1}\left(S^{1}, \mathbf{R}^{2}\right),\langle\cdot, \cdot\rangle$ and $\|\cdot\|$ can be explicitly expressed by

$$
\langle z, y\rangle=\int_{0}^{2 \tau}\left[(z(t), y(t))+\left(z^{\prime}(t), y^{\prime}(t)\right)\right] d t
$$

and

$$
\|z\|=\left[\left|a_{0}\right|^{2}+\sum_{j=1}^{\infty}\left(1+j^{2}\right)\left(\left|a_{j}\right|^{2}+\left|b_{j}\right|^{2}\right)\right]^{1 / 2} .
$$

We define an operator $L: H^{1}\left(S^{1}, \mathbf{R}^{2}\right) \rightarrow H^{1}\left(S^{1}, \mathbf{R}^{2}\right)$ by the Riesz representation theorem

$$
\langle L z, y\rangle=\int_{0}^{2 \tau}(\dot{z}(t), \dot{y}(t)) d t-\lambda \int_{0}^{2 \tau}(z(t), y(t)) d t
$$

By a direct computation, $L$ is a bounded self-adjoint linear operator on $H^{1}\left(S^{1}, \mathbf{R}^{2}\right)$. 
By $(f 2)$ and (3.6) in Section 3, there exist two continuous functions $\alpha(t)>0$ and $\beta(t) \geq 0$ such that

$$
H(t, z) \leq \alpha(t)|z|^{2}+\beta(t), \quad \forall z \in \mathbf{R}^{2} .
$$

By using similar arguments as in [3], we have that

$$
\varphi(z)=\frac{1}{2}\langle L z, z\rangle+\psi(z)
$$

where

$$
\psi(z)=\int_{0}^{2 \tau} H(t, z(t)) d t
$$

Thus the critical points of $\varphi(z)$ in $H^{1}\left(S^{1}, \mathbf{R}^{2}\right)$ are classical solutions of (2.2).

Denote $E=\left\{z=(x, y) \in H^{1}\left(S^{1}, \mathbf{R}^{2}\right): y(t)=x(t-\tau)\right\}$. By a direct computation, we have

$$
\begin{aligned}
E= & \left\{z \in H^{1}\left(S^{1}, \mathbf{R}^{2}\right) \mid z(t)=\frac{1}{\sqrt{2 \tau}} a_{0}\left(\begin{array}{l}
1 \\
1
\end{array}\right)\right. \\
& \left.+\frac{1}{\sqrt{\tau}} \sum_{j=1}^{\infty}\left(a_{j} \cos \left(\frac{\pi}{\tau} j t\right)+b_{j} \sin \left(\frac{\pi}{\tau} j t\right)\right)\left(\begin{array}{c}
1 \\
(-1)^{j}
\end{array}\right)\right\},
\end{aligned}
$$

where $a_{0}, a_{j}, b_{j} \in \mathbf{R}, j=1,2, \ldots$

Note that for any $z=(x, y) \in E, y(t)=x(t-\tau)$ and $\nabla H(t, z)=(f(t, x, y), f(t, y, x))$. Due to the fact that $f(t, x(t-\tau), y(t-\tau))=f(t, y(t), x(t))$, we know that $\nabla H(t, z) \in E$ whatever $z \in E$. Therefore, we have the following lemma.

Lemma 2.3 If $z(t)$ is a critical point of $\varphi$ in $E$, then $z(t)$ is a critical point of $\varphi$ in $H^{1}\left(S^{1}, \mathbf{R}^{2}\right)$.

Moreover, we also denote by $M^{+}(\cdot), M^{-}(\cdot)$ and $M^{0}(\cdot)$ the positive definite, negative definite and null subspaces of the self-adjoint linear operator defining it, respectively.

Then $E$ has an orthogonal decomposition

$$
E=M^{+}(L) \oplus M^{-}(L) \oplus M^{0}(L) .
$$

Let

$$
z_{j}(t)=\frac{1}{\sqrt{\tau}}\left(a_{j} \cos \left(\frac{\pi}{\tau} j t\right)+b_{j} \sin \left(\frac{\pi}{\tau} j t\right)\right)\left(\begin{array}{c}
1 \\
(-1)^{j}
\end{array}\right), \quad j=1,2, \ldots,
$$

where $a_{j}, b_{j} \in \mathbf{R}$. We have

$$
\left\langle L z_{j}, z_{j}\right\rangle=\frac{1}{1+j^{2}}\left(\frac{\pi^{2}}{\tau^{2}} j^{2}-\lambda\right)\left\|z_{j}\right\|, \quad j=1,2, \ldots
$$

There exists $\sigma>0$ such that

$$
\left\langle L z_{j}, z_{j}\right\rangle \geq \sigma\left\|z_{j}\right\|^{2} \quad \text { for } j \in J^{+},
$$




$$
\left\langle L z_{j}, z_{j}\right\rangle \leq-\sigma\left\|z_{j}\right\|^{2} \quad \text { for } j \in J^{-}
$$

where $J^{+}=\left\{j \geq 1: \frac{1}{1+j^{2}}\left(\frac{\pi^{2}}{\tau^{2}} j^{2}-\lambda\right)>0\right\}, J^{-}=\left\{j \geq 1: \frac{1}{1+j^{2}}\left(\frac{\pi^{2}}{\tau^{2}} j^{2}-\lambda\right)<0\right\}, J^{0}=\left\{j \geq 1: \frac{1}{1+j^{2}}\left(\frac{\pi^{2}}{\tau^{2}} j^{2}-\right.\right.$ $\lambda)=0\}$. Therefore, we get

$$
\begin{aligned}
& \langle L z, z\rangle \geq \sigma\|z\|^{2} \quad \text { for } z \in M^{+}(L), \\
& \langle L z, z\rangle \leq-\sigma\|z\|^{2} \quad \text { for } z \in M^{-}(L) .
\end{aligned}
$$

$\operatorname{Remark} 2.1$ The condition $\lambda>\pi^{2} / \tau^{2}$ is to ensure $J^{-} \neq \emptyset$.

\section{Proofs of theorems}

In this section, $c_{i}$ stand for different positive constants for $i \in \mathbf{Z}^{+}$.

By a direct computation, $(f 1)$ and $(f 2)$ imply that $H(t, z)$ is even and satisfies

$$
\begin{aligned}
& \nabla H(t, z)=A(t) z+\circ(|z|) \quad \text { as }|z| \rightarrow 0, \\
& \nabla H(t, z)=B(t) z+\circ(|z|) \quad \text { as }|z| \rightarrow \infty
\end{aligned}
$$

uniformly for $t \in[0, \tau]$.

$\left(f 3^{ \pm}\right)$implies that

$$
\begin{aligned}
& |\nabla H(t, z)-B(t) z| \quad \text { is bounded and } \\
& H(t, z)-\frac{1}{2}(B(t) z, z) \rightarrow \pm \infty \quad \text { as }|z| \rightarrow \infty
\end{aligned}
$$

uniformly for $t \in[0, \tau]$.

Lemma 3.1 Suppose that $f$ satisfies $(f 1)-\left(f 3^{ \pm}\right)$. Then the function $\varphi$ satisfies the $(C)_{c}$ condition for any $c \in \mathbf{R}$.

Proof First we define an operator

$$
\langle B z, y\rangle=\int_{0}^{2 \tau}(B(t) z(t), y(t)) d t
$$

for any $z, y \in E$. By a direct computation, $B$ is a bounded self-adjoint linear operator on $E$. Thus $L+B$ is also a self-adjoint linear operator on $E$. Then $E$ has an orthogonal decomposition

$$
E=M^{+}(L+B) \oplus M^{-}(L+B) \oplus M^{0}(L+B) .
$$

Also there exists $\sigma_{1}>0$ such that

$$
\begin{aligned}
& \langle(L+B) z, z\rangle \geq \sigma_{1}\|z\|^{2} \quad \text { for } z \in M^{+}(L+B), \\
& \langle(L+B) z, z\rangle \leq-\sigma_{1}\|z\|^{2} \quad \text { for } z \in M^{-}(L+B) .
\end{aligned}
$$


So, set

$$
\Phi(z)=-\varphi(z)=-\frac{1}{2}\langle(L+B) z, z\rangle-\int_{0}^{2 \tau}\left[H(t, z)-\frac{1}{2}(B(t) z, z)\right] d t
$$

and

$$
\left\langle\Phi^{\prime}(z), y\right\rangle=-\langle(L+B) z, y\rangle-\int_{0}^{2 \tau}(\nabla H(t, z)-B(t) z, y) d t
$$

for any $z, y \in E, \varphi(z)$ is defined in Section 2.

Let $\left\{z_{k}\right\} \subset E$ be any sequence such that

$$
\Phi\left(z_{k}\right) \rightarrow c, \quad\left(1+\left\|z_{k}\right\|\right) \Phi^{\prime}\left(z_{k}\right) \rightarrow 0 \quad \text { as } k \rightarrow \infty .
$$

We first prove that $\left\{z_{k}\right\}$ is bounded. For any $\varepsilon>0$, (3.2) implies that there exists a constant $C_{\varepsilon}>0$ such that

$$
|\nabla H(t, z)-B(t) z|<\varepsilon|z|+C_{\varepsilon}
$$

for all $z \in \mathbf{R}^{2}$ and $t \in[0,2 \tau]$. Since $z_{k} \in E$, we have

$$
z_{k}=z_{k}^{+}+z_{k}^{-}+z_{k}^{0}
$$

where $z_{k}^{+} \in M^{+}(L+B), z_{k}^{-} \in M^{-}(L+B), z_{k}^{0} \in M^{0}(L+B)$. By $\left(3.3^{ \pm}\right)$, there exists a constant $d>0$ such that

$$
|\nabla H(t, z)-B(t) z| \leq d
$$

Therefore we have

$$
\begin{aligned}
(1+c)\left\|z_{k}^{+}\right\| & \geq\left\langle-\Phi^{\prime}\left(z_{k}\right), z_{k}^{+}\right\rangle \\
& =\left\langle(L+B) z_{k}^{+}, z_{k}^{+}\right\rangle+\int_{0}^{2 \tau}\left(\nabla H\left(t, z_{k}\right)-B(t) z_{k}, z_{k}^{+}\right) d t \\
& \geq \sigma_{1}\left\|z_{k}^{+}\right\|^{2}-d\left\|z_{k}^{+}\right\| .
\end{aligned}
$$

Thus we have that $\left\{\left\|z_{k}^{+}\right\|\right\}$is bounded. Using similar arguments, we can prove that $\left\{\left\|z_{k}^{-}\right\|\right\}$ is bounded. Consider $\left\{\left\|z_{k}^{0}\right\|\right\}$. Arguing indirectly, we suppose $\left\{\left\|z_{k}^{0}\right\|\right\}$ is unbounded, then we have $\left\|z_{k}^{0}\right\| \rightarrow \infty$. According to the definition of $M^{0}(L+B)$, this implies that there are constants $d_{1}, d_{2}>0$ such that

$$
d_{1}\left|z_{k}^{0}\right| \leq\left\|z_{k}^{0}\right\| \leq d_{2}\left|z_{k}^{0}\right|
$$

By (3.7), we have

$$
\left|z_{k}\right| \geq\left|z_{k}^{0}\right| \rightarrow+\infty \quad \text { as }\left\|z_{k}^{0}\right\| \rightarrow \infty
$$


Then

$$
\begin{aligned}
2 c= & \lim _{k \rightarrow \infty}\left[2 \Phi\left(z_{k}\right)-\Phi^{\prime}\left(z_{k}\right) z_{k}\right] \\
= & \lim _{k \rightarrow \infty}\left\{\int_{0}^{2 \tau}\left(\nabla H\left(t, z_{k}\right)-B(t) z_{k}, z_{k}\right) d t\right. \\
& \left.-2 \int_{0}^{2 \tau}\left[H\left(t, z_{k}\right)-\frac{1}{2}\left(B(t) z_{k}, z_{k}\right)\right] d t\right\} .
\end{aligned}
$$

By (3.8) and $\left(3.3^{ \pm}\right),(3.9)$ is a contradiction. Hence $\left\{\left\|z_{k}^{0}\right\|\right\}$ is bounded. Therefore there exists a constant $d_{3}>0$ such that

$$
\left\|z_{k}\right\|=\left\|z_{k}^{+}\right\|+\left\|z_{k}^{-}\right\|+\left\|z_{k}^{0}\right\| \leq d_{3} .
$$

So, we get $\left\{z_{k}\right\}$ is bounded, and going if necessary to a subsequence, we can assume that $z_{k} \rightarrow z$ in $E$ and $z_{k} \rightarrow z$ in $C\left(S^{1}, \mathbf{R}^{N}\right)$. Write $z_{k}=z_{k}^{+}+z_{k}^{-}+z_{k}^{0}$ and $z=z^{+}+z^{-}+z^{0}$, then $z_{k}^{ \pm} \rightarrow z^{ \pm}$in $E, z_{k}^{0} \rightarrow z^{0}$ in $E$ and $z_{k}^{ \pm} \rightarrow z^{ \pm}$in $C\left(S^{1}, \mathbf{R}^{N}\right)$.

In view of (3.6) and $z_{k}^{-} \rightarrow z^{-}$in $C\left(S^{1}, \mathbf{R}^{2}\right)$, it is easy to verify

$$
\int_{0}^{2 \tau}\left(\nabla H\left(t, z_{k}\right)-B(t) z_{k}, z_{k}^{-}-z^{-}\right) d t \rightarrow 0
$$

and

$$
\int_{0}^{2 \tau}\left(\nabla H(t, z)-B(t) z, z_{k}^{-}-z^{-}\right) d t \rightarrow 0 .
$$

But then $\left\langle\Phi^{\prime}\left(z_{k}\right)-\Phi^{\prime}(z), z_{k}^{-}-z^{-}\right\rangle \rightarrow 0$ as $k \rightarrow \infty$, and

$$
\begin{aligned}
& \left\langle\Phi^{\prime}\left(z_{k}\right)-\Phi^{\prime}(z), z_{k}^{-}-z^{-}\right\rangle \\
& =-\left\langle(L+B)\left(z_{k}^{-}-z^{-}\right), z_{k}^{-}-z^{-}\right\rangle \\
& \quad-\int_{0}^{2 \tau}\left(\nabla H\left(t, z_{k}\right)-B(t) z_{k}, z_{k}^{-}-z^{-}\right) d t \\
& \quad+\int_{0}^{2 \tau}\left(\nabla H(t, z)-B(t) z, z_{k}^{-}-z^{-}\right) d t \\
& \geq \sigma_{1}\left\|z_{k}^{-}-z^{-}\right\|-\int_{0}^{2 \tau}\left(\nabla H\left(t, z_{k}\right)-B(t) z_{k}, z_{k}^{-}-z^{-}\right) d t \\
& \quad+\int_{0}^{2 \tau}\left(\nabla H(t, z)-B(t) z, z_{k}^{-}-z^{-}\right) d t .
\end{aligned}
$$

This yields $z_{k}^{-} \rightarrow z^{-}$in $E$. Similarly, $z_{k}^{+} \rightarrow z^{+}$in $E$ and hence $z_{k} \rightarrow z$ in $E$, that is, $\Phi$ satisfies the $(C)_{c}$ condition. Thus $\varphi$ satisfies the $(C)_{c}$ condition. The proof of Lemma 3.1 is complete.

Proof of Theorem 1.1 For $z \in E$, let $X=M^{+}(L) \oplus M^{0}(L), Y=M^{-}(L), E=X \oplus Y$, and

$$
\Phi(z)=-\varphi(z), \quad \Psi(z)=\psi(z)
$$

where $\varphi(z)$ and $\psi(z)$ are from Section 2 . 
In order to obtain this theorem, we apply Theorem A to the functional $\Phi(z)$. The proof of this theorem is divided into the following three steps.

Step 1. $\Phi$ satisfies $\left(\Phi_{0}\right)$.

We first check that $\Phi_{c}$ is $\mathcal{T}_{\mathcal{S}}$-closed for any $c \in \mathbf{R}$. Let $\left\{z_{k}\right\}$ be any sequence $\mathcal{T}_{\mathcal{S}}$ converging to some $z \in E$. Write $z_{k}=z_{k}^{+}+z_{k}^{0}+z_{k}^{-}$and $z=z^{+}+z^{0}+z^{-}$, then $z_{k}^{-} \rightarrow u^{-}$in $E$ and hence $\left\{z^{-}\right\}$is bounded in the norm topology. Note that $(B(t) z, z)>\lambda-\pi^{2} / \tau^{2}>0$ for $z \in \mathbf{R}^{2} \backslash\{0\}$ and for $t \in[0,2 \tau]$, then for any $z \in E$ and small $\varepsilon>0$, we have by (3.10) and (3.6)

$$
\begin{aligned}
\Psi(z) & =\int_{0}^{2 \tau} H(t, z) d t \\
& =\int_{0}^{2 \tau} \int_{0}^{1}(\nabla H(t, s z), z) d s d t \\
& \geq \int_{0}^{2 \tau} \int_{0}^{1}(B(t) s z, z) d s d t-\int_{0}^{2 \tau} \int_{0}^{1}|\nabla H(t, z)-(B(t) s z, z)||z| d s d t \\
& \geq \frac{1}{2} \int_{0}^{2 \tau}(B(t) z, z) d t-\int_{0}^{2 \tau} \int_{0}^{1}\left(\varepsilon|s z|+C_{\varepsilon}\right)|z| d s d t \\
& \geq \frac{1}{2}\left(\lambda-\frac{\pi^{2}}{\tau^{2}}-\varepsilon\right)\|z\|_{L^{2}}^{2}-C_{\varepsilon}\|z\|_{L^{2}} \geq c_{1}\|z\|_{L^{2}}^{2}-c_{2}\|z\|_{L^{2}},
\end{aligned}
$$

which implies that $\Psi$ is bounded from below on $E$. Consequently, combining $z_{k} \in \Phi_{c}$ and $\frac{1}{2}\left\langle L z_{k}^{+}, z_{k}^{+}\right\rangle=-\frac{1}{2}\left\langle L z_{k}^{-}, z_{k}^{-}\right\rangle-\Phi\left(z_{k}\right)-\Psi\left(z_{k}\right)$ shows that $\left\{z_{k}^{+}\right\}$is bounded in $E$ by (2.3) and hence

$$
\begin{aligned}
\Psi\left(z_{k}\right) & =-\frac{1}{2}\left\langle L z_{k}^{+}, z_{k}^{+}\right\rangle-\frac{1}{2}\left\langle L z_{k}^{-}, z_{k}^{-}\right\rangle-\Phi\left(z_{k}\right) \\
& \leq-\frac{1}{2}\left\langle L z_{k}^{+}, z_{k}^{+}\right\rangle-\frac{1}{2}\left\langle L z_{k}^{-}, u_{k}^{-}\right\rangle-c \leq c_{3} .
\end{aligned}
$$

Moreover, since $\left\|z_{k}\right\|_{L^{2}}^{2}=\left\|z_{k}^{+}\right\|_{L^{2}}^{2}+\left\|z_{k}^{0}\right\|_{L^{2}}^{2}+\left\|z_{k}^{-}\right\|_{L^{2}}^{2}$, we have

$$
\Psi\left(z_{k}\right) \geq c_{1}\left\|z_{k}\right\|_{L^{2}}^{2}-c_{2}\left\|z_{k}\right\|_{L^{2}} \geq\left\|z_{k}\right\|_{L^{2}}-c_{4} \geq\left\|z_{k}^{0}\right\|_{L^{2}}-c_{4} .
$$

It follows from (3.11) and the above inequality that $\left\{z_{k}^{0}\right\}$ is also bounded in $E$ since all norms are equivalent in a finite dimensional space. Then $\left\|z_{k}\right\|^{2}\left(=\left\|z_{k}^{+}\right\|^{2}+\left\|z_{k}^{0}\right\|^{2}+\left\|z_{k}^{-}\right\|^{2}\right)$ is bounded, and hence we can assume that $\left\{z_{k}\right\}$ converges weakly to $z=z^{+}+z^{0}+z^{-}$in $E$. Thus we have $\Psi\left(z_{k}\right) \rightarrow \Psi(z)$. Note that $\langle L y, y\rangle^{1 / 2}$ is an equivalent norm on $H^{+}$. By the lower semi-continuity of the norm, we get

$$
\begin{aligned}
c & \leq \limsup _{k \rightarrow \infty} \Phi\left(z_{k}\right)=\limsup _{k \rightarrow \infty}\left(-\frac{1}{2}\left\langle L z_{k}^{+}, z_{k}^{+}\right\rangle-\frac{1}{2}\left\langle L z_{k}^{-}, z_{k}^{-}\right\rangle-\Psi\left(z_{k}\right)\right) \\
& =-\liminf _{k \rightarrow \infty} \frac{1}{2}\left\langle L z_{k}^{+}, z_{k}^{+}\right\rangle-\frac{1}{2}\left\langle L z^{-}, z^{-}\right\rangle-\Psi(z) \leq \Phi(z),
\end{aligned}
$$

that is, $z \in \Phi_{c}$ and hence $\Phi_{c}$ is $\mathcal{T}_{\mathcal{S}}$-closed.

Next, we prove that $\Phi^{\prime}:\left(\Phi_{c}, \mathcal{T}_{\mathcal{S}}\right) \rightarrow\left(E^{*}, \omega^{*}\right)$ is continuous. To achieve this, it is sufficient to demonstrate that $\Psi^{\prime}$ has the same property. Suppose $z_{k} \rightarrow u$ in $E$, then $\left\{z_{k}\right\}$ converges uniformly to $z$ on $[0,2 \tau]$. Hence, for every given $y \in E$, we see that $\left(\nabla H\left(t, z_{k}(t)\right), y(t)\right)$ con- 
verges to $(\nabla H(t, z(t)), y(t))$ in measure on $[0,2 \tau]$. Moreover, by (3.6), one has

$$
\left.\mid \nabla H\left(t, z_{k}(t)\right), y(t)\right) \mid \leq\left(\varepsilon\left\|z_{k}\right\|_{\infty}+\bar{b}\left\|z_{k}\right\|_{\infty}+C_{\varepsilon}\right)\|y\|_{\infty} \leq c_{5}
$$

for all $k$ and $t \in[0,2 \tau]$, where $\bar{b}=\max _{t \in[0, \tau]}\{|B(t)|\}$ and $\|\cdot\|_{\infty}$ denotes the natural norm of $C\left(S^{1}, \mathbf{R}^{2}\right)$. Thus, the Vitali theorem is applicable and

$$
\left\langle\Psi^{\prime}\left(z_{k}\right), y\right\rangle=\int_{0}^{2 \tau}\left(\nabla H\left(t, z_{k}\right), y\right) d t \rightarrow \int_{0}^{2 \tau}(\nabla H(t, z), y) d t=\left\langle\Psi^{\prime}(z), y\right\rangle
$$

for any $y \in E$. So, $\Phi$ satisfies $\left(\Phi_{0}\right)$.

Step 2. $\Phi$ satisfies $\left(\Phi_{1}\right)$.

By (3.1), there exist $0<\varepsilon<\sigma / 2, \rho_{0}>0$ such that

$$
H(t, z) \leq \varepsilon|z|^{2}+\frac{1}{2}(A(t) z, z) \quad \text { for all }|z|<\rho_{0}, \quad t \in[0,2 \tau] .
$$

By Proposition 1.1 in [3], there is a positive constant $\xi$ such that $\|z\|_{\infty} \leq \xi\|z\|$. Set small $\rho<\rho_{0} / \xi$, then for each $z \in Y$ with $\|z\| \leq \rho$, one has $\|z\|_{\infty} \leq \rho_{0}$, and hence by (3.12)

$$
\begin{aligned}
\Phi(z) & =-\frac{1}{2}\langle L z, z\rangle-\int_{0}^{2 \tau} H(t, z) d t \\
& \geq \frac{\sigma}{2}\|z\|^{2}-\int_{0}^{2 \tau}\left[\varepsilon|z|^{2}+\frac{1}{2}(A(t) z, z)\right] d t \geq\left(\frac{\sigma}{2}-\varepsilon\right)\|z\|^{2} .
\end{aligned}
$$

Therefore,

$$
\kappa:=\inf \Phi\left(B_{\rho} \cap Y\right) \geq\left(\frac{\sigma}{2}-\varepsilon\right) \rho^{2}>0,
$$

and hence $\left(\Phi_{1}\right)$ holds.

Step 3. $\Phi$ satisfies $\left(\Phi_{2}\right)$.

Let

$$
Y_{0}=\operatorname{span}\left\{\cos \left(\frac{\pi}{\tau} j t\right)\left(\begin{array}{c}
1 \\
(-1)^{j}
\end{array}\right), \sin \left(\frac{\pi}{\tau} j t\right)\left(\begin{array}{c}
1 \\
(-1)^{j}
\end{array}\right): j \in \mathbf{Z}^{+}, j \leq m\right\} .
$$

Obviously, $Y_{0} \subset Y$ and $\operatorname{dim} Y_{0}=2 m$. In order to obtain the desired conclusion, it is sufficient to prove that $\Phi(z) \rightarrow-\infty$ as $\|z\| \rightarrow \infty$ on $E_{0}:=X \oplus Y_{0}$.

Let $\Gamma=\lambda-\frac{\pi^{2}}{\tau^{2}}$. By the definition of $m$, there exists a constant $0<\delta<\sigma$ such that

$$
B(t) \geq \Gamma+\delta
$$

for $t \in[0, \tau]$. Clearly, for any $y \in Y_{0}$,

$$
\langle L y, y\rangle \geq-\Gamma\|y\|_{L^{2}}^{2} .
$$

Let $\widetilde{F}(t, z)=H(t, z)-\frac{1}{2}(B(t) z, z)$. We claim that

$$
\|z\|^{-2} \int_{0}^{2 \tau} \widetilde{F}(t, z) d t \rightarrow 0 \quad \text { as }\|z\| \rightarrow \infty .
$$


Indeed, for $0 \neq z \in E$, by (3.6), one has

$$
\begin{aligned}
& \|z\|^{-2}\left|\int_{0}^{2 \tau} \widetilde{F}(t, z) d t\right| \\
& =\|z\|^{-2}\left|\int_{0}^{2 \tau} \int_{0}^{1}(\nabla H(t, s z)-B(t) s z, z) d s d t\right| \\
& \leq\|z\|^{-2} \int_{0}^{2 \tau}\left(\varepsilon|z|+C_{\varepsilon}\right)|z| d t \\
& \leq\|z\|^{-2}\left(\varepsilon\|z\|_{L^{2}}^{2}+C_{\varepsilon}\|z\|_{L^{2}}\right) \\
& \quad \leq \varepsilon+\frac{C_{\varepsilon}}{\|z\|}
\end{aligned}
$$

which implies that (3.15) is true by the arbitrariness of $\varepsilon$. Then, for $z=z^{+}+z^{0}+z^{-} \in E_{0}$, by (2.3), (3.13) and (3.14), one has

$$
\begin{aligned}
\Phi(z) & =-\frac{1}{2}\left\langle L z^{-}, z^{-}\right\rangle-\frac{1}{2}\left\langle L z^{+}, z^{+}\right\rangle-\int_{0}^{2 \tau} F(t, z) d t \\
& \leq \frac{\Gamma}{2}\left\|z^{-}\right\|_{L^{2}}^{2}-\frac{\delta}{2}\left\|z^{+}\right\|^{2}-\frac{1}{2} \int_{0}^{2 \tau}(B(t) z, z) d t-\int_{0}^{2 \tau} \widetilde{F}(t, z) d t \\
& \leq \frac{\Gamma}{2}\left\|z^{-}\right\|_{L^{2}}^{2}-\frac{\delta}{2}\left\|z^{+}\right\|^{2}-\frac{1}{2}(\Gamma+\delta)\|z\|_{L^{2}}^{2}-\int_{0}^{2 \tau} \widetilde{F}(t, z) d t \\
& \leq \frac{\Gamma}{2}\left\|z^{-}\right\|_{L^{2}}^{2}-\frac{\delta}{2}\left\|z^{+}\right\|^{2}-\frac{1}{2}(\Gamma+\delta)\left(\left\|z^{-}\right\|_{L^{2}}^{2}+\left\|z^{0}\right\|_{L^{2}}^{2}\right)-\int_{0}^{2 \tau} \widetilde{F}(t, z) d t \\
& \leq-\frac{\delta}{2}\left(\left\|z^{-}\right\|_{L^{2}}^{2}+\left\|z^{+}\right\|^{2}+\left\|z^{0}\right\|_{L^{2}}^{2}\right)-\int_{0}^{2 \tau} \widetilde{F}(t, z) d t .
\end{aligned}
$$

Since $M^{0}$ and $Y_{0}$ are finitely dimensional, (3.15) and the above estimate imply that $\Phi(z) \rightarrow$ $-\infty$ as $\|z\| \rightarrow \infty, z \in E_{0}:=X \oplus Y_{0}$. Hence $\left(\Phi_{2}\right)$ holds.

The proof of Theorem 1.1 is complete.

Proof of Theorem 1.2 For $z \in E$, let $X=M^{-}(L) \oplus M^{0}(L), Y=M^{+}(L), E=X \oplus Y$, and

$$
\Phi(z)=\varphi(z), \quad \Psi(z)=-\psi(z)
$$

and

$$
Y_{0}=\operatorname{span}\left\{\cos \left(\frac{\pi}{\tau} j t\right)\left(\begin{array}{c}
1 \\
(-1)^{j}
\end{array}\right), \sin \left(\frac{\pi}{\tau} j t\right)\left(\begin{array}{c}
1 \\
(-1)^{j}
\end{array}\right): j=m_{0}, m_{0}+1, \ldots, m_{1}\right\} .
$$

Then the conclusion is obtained by the same argument as in the proof of Theorem 1.1. The proof of Theorem 1.2. is complete.

Example 3.1 Consider the following equation:

$$
x^{\prime \prime}(t)+\lambda x(t)=-\alpha(t) x(t)-\beta(t) x(t-\pi)-\frac{x(t)}{x^{2}(t)+x^{2}(t-\pi)+\gamma^{2}(t)},
$$


where $x(t) \geq 0$. Let

$$
f(t, x, y)=\alpha(t) x+\beta(t) y+\frac{x}{x^{2}+y^{2}+\gamma^{2}(t)} .
$$

Then

$$
\frac{\partial f(t, x, y)}{\partial y}=\beta(t)-\frac{2 x y}{\left(x^{2}+y^{2}+\gamma^{2}(t)\right)^{2}}=\frac{\partial f(t, y, x)}{\partial x} .
$$

Let

$$
H(t, x, y)=\frac{1}{2} \alpha(t) x^{2}+\beta(t) x y+\frac{1}{2} \alpha(t) y^{2}+\frac{1}{2} \ln \frac{x^{2}+y^{2}+\gamma^{2}(t)}{\gamma^{2}(t)} .
$$

Then

$$
\frac{\partial H}{\partial x}=f(t, x, y), \quad \frac{\partial H}{\partial y}=f(t, y, x) .
$$

By a straightforward computation, we have

$$
f(t, x, y)=\left(\frac{1}{\gamma^{2}(t)}+\alpha(t)\right) x+\beta(t) y+o(r) \quad \text { as } r=\sqrt{x^{2}+y^{2}} \rightarrow 0
$$

and

$$
f(t, x, y)=\alpha(t) x+\beta(t) y+o(r) \quad \text { as } r=\sqrt{x^{2}+y^{2}} \rightarrow \infty
$$

uniformly for $t \in[0, \pi]$.

Take $\lambda=6, \alpha(t)=-5+\sin \pi t, \beta(t)=1-\sin \pi t, \gamma^{2}(t)=\frac{1}{6}$. By Theorem 1.2, we have $a(t)=$ $\frac{1}{\gamma^{2}(t)}+\alpha(t) \geq 0, b(t)=d(t)=1-\sin \pi t \geq 0, c(t)=-5+\sin \pi t$ for $t \in[0, \pi], z=(x, y), x \geq 0$, $y \geq 0$,

$$
\begin{aligned}
(A(t) z, z) & =\left(\left(\begin{array}{ll}
a(t) & b(t) \\
b(t) & a(t)
\end{array}\right)\left(\begin{array}{l}
x \\
y
\end{array}\right),\left(\begin{array}{l}
x \\
y
\end{array}\right)\right) \geq 0, \\
(B(t) z, z) & =\left(\left(\begin{array}{ll}
c(t) & d(t) \\
d(t) & c(t)
\end{array}\right)\left(\begin{array}{l}
x \\
y
\end{array}\right),\left(\begin{array}{l}
x \\
y
\end{array}\right)\right) \\
& =c(t)\left(x^{2}+y^{2}\right)+2 d(t) x y \leq[c(t)+d(t)]\left(x^{2}+y^{2}\right) \\
& =-4\left(x^{2}+y^{2}\right)<\left(6-3^{2}\right)\left(x^{2}+y^{2}\right)<0 .
\end{aligned}
$$

Then we have $m_{0}=m_{1}=3$. By Theorem 1.2, (3.16) possesses at least two pairs $2 \pi$-periodic solutions.

The authors declare that they have no competing interests. 


\section{Author details}

'School of Mathematical Science, Shanxi University, Wucheng Road, Taiyuan, 030006, P.R. China. ${ }^{2}$ School of Mathematical Sciences, Capital Normal University, Beijing, 100048, P.R. China.

\section{Acknowledgements}

The authors are grateful for the referees' careful reviewing and their valuable suggestions. The work is partially supported by the Natural Science Foundation of Shanxi Province (No. 2012011004-1) of China.

\section{Received: 18 May 2013 Accepted: 12 September 2013 Published: 19 Nov 2013}

\section{References}

1. Ding, Y: Variational Methods for Strongly Indefinite Problems. World Scientific, Singapore (2007)

2. Guo, Y: Nontrivial periodic solutions for asymptotically linear Hamiltonian systems with resonance. J. Differ. Equ. 175, 71-87 (2001)

3. Mawhin, J, Willem, M: Critical Point Theory and Hamiltonian Systems. Springer, New York (1989)

4. Rabinowits, PH: Minimax Methods in Critical Point Theory with Applications to Differential Equations, CBMS American Mathematical Society, vol. 65 (1986)

5. Su, JB: Nontrivial periodic solutions for the asymptotically linear Hamiltonian systems with resonance at infinity. J. Differ. Equ. 145, 252-273 (1998)

6. Zhao, F, Zhao, L, Ding, Y: Existence and multiplicity of solutions for a non-periodic Schrödinger equation. Nonlinear Anal. TMA 69, 3671-3678 (2008)

7. Li, J, He, X: Multiple periodic solutions of differential delay equations created by asymptotically linear Hamiltonian systems. Nonlinear Anal. 31(1/2), 45-54 (1998)

8. Guo, Z, Yu, J: Multiplicity results for periodic solutions to delay differential equations via critical point theory. J. Differ. Equ. 218, 15-35 (2005)

9. Fei, G: Multiple periodic solutions of differential delay equations via Hamiltonian systems (I). Nonlinear Anal. 65, 25-39 (2006)

10. Fei, G: Multiple periodic solutions of differential delay equations via Hamiltonian systems (II). Nonlinear Anal. 65 40-58 (2006)

11. Guo, Z, Xiaomin, Z: Multiple results for periodic solutions to a class of second-order delay differential equations Commun. Pure Appl. Anal. 9(6), 1529-1542 (2010)

12. Wu, K, Wu, X, Zhou, F: Multiplicity results of periodic solutions for a class of second order delay differential equations. Nonlinear Anal. 75, 5836-5844 (2012)

13. Yu, J, Xiao, H: Multiplicity periodic solutions with minimal period 4 of delay differential equations $x^{\prime}(t)=-f(t, x(t-1))$. J. Differ. Equ. 254, 2158-2172 (2013)

14. Zhang, X, Meng, Q: Nontrivial periodic solutions for delay differential systems via Morse theory. Nonlinear Anal. 74, 1960-1968 (2011)

10.1186/1687-2770-2013-244

Cite this article as: Meng and Zhang: Multiple periodic solutions to a class of nonautonomous second-order delay differential equation. Boundary Value Problems 2013, 2013:244

\section{Submit your manuscript to a SpringerOpen ${ }^{\ominus}$ journal and benefit from:}

- Convenient online submission

- Rigorous peer review

Immediate publication on acceptance

- Open access: articles freely available online

- High visibility within the field

- Retaining the copyright to your article 